\title{
Lie subalgebras of finite codimension of the classical infinite Lie algebras
}

\author{
By \\ Nobutada NAKANISHI \\ (Communicated by Prof. H. Toda, Feb. 20, 1978)
}

\section{Introduction}

The present paper is a sequel of the author's papers [6] and [7]. The structure of some ideals of the global primitive infinite Lie algebras was studied in [5]. In this paper we will study the structure of Lie subalgebras of finite codimension of the classical infinite Lie algebras. We mean by a classical infinite Lie algebra one of the following Lie algebras:

(1) the Lie algebra of all vector fields,

(2) the Lie algebra of vector fields of divergence zero,

(3) the Lie algebra of vector fields preserving a hamiltonian structure (the hamiltonian Lie algebra),

(4) the Lie algebra of vector fields preserving a contact structure (the contact Lie algebra).

In $\S 1$, we define the classical infinite Lie algebras from both formal and global versions. A formal classical infinite Lie algebra $L$ is topologized by its natural filtration $\left\{L_{p}\right\}_{p \in Z}$ (see $\S 2$ ).

Our main theorem is then stated as follows:

Theorem A. Let $L$ be a formal classical infinite Lie algebra. If $B$ is a proper Lie subalgebra of finite codimension of $L$, then there exists a positive integer $k$ such that $L_{k} \subset B \subset L_{0}$. (This means that $B$ is a closed Lie subalgebra of L.)

Using the above theorem, as an immediate consequence, we can get the following (see $\S 7)$.

Theorem B. Let $\mathscr{L}$ be a global classical infinite Lie algebra. (That is, $\mathscr{L}$ is defined on a differentiable manifold $M$.) If $\mathscr{B}$ is a Lie subalgebra of finite codimension of $\mathscr{L}$, then it holds either a) or b).

a) $B_{x}=L_{x}$ for all $x \in M$, where $B_{x}$ (resp. $L_{x}$ ) is the formal algebra of $\mathscr{B}$ (resp. $\mathscr{L})$ at $x$. In particular, $\mathscr{B}$ is transitive at every point $x \in M$.

b) There exist a finite number of points $a_{1}, \cdots, a_{r}$ of $M$ such that 


$$
\mathscr{B} \subset \bigcap_{i=1}^{r}\left\{X \in \mathscr{L} ; X\left(a_{i}\right)=0\right\} .
$$

In $\S 2$, we list up the well-known properties of the formal classical infinite Lie algebras. Recently K. Masuda [2] proved the result for the Lie algebra of all global vector fields. The principle of the proof of our main theorem is partly similar to that of [2], but we will make full use of the properties of the formal classical infinite Lie algebras.

In $\S 3$, we prove the main theorem for the Lie algebra of all formal vector fields.

For the other formal classical infinite Lie algebras, we need some important lemmata. These are proved in $\S 4,5$ and 6 . Then the proof of the main theorem is carried out in $\S 7$.

In $\S 8$, we give an example of an infinite Lie algebra $L$ which has a Lie subalgebra $B$ of finite codimension such that $B \measuredangle L_{0}$.

The author would like to express his gratitude to Professors N. Tanaka and T. Morimoto for their constant encouragements.

\section{§1. Classical infinite Lie algebras}

The following Lie algebras are called the formal classical infinite Lie algebras:

(1) $L_{g l}(n)$ : the Lie algebra of all formal (or better, formal power series) vector fields in $n$-variables $x_{1}, \cdots, x_{n}$.

(2) $L_{s l}(n)$ : the Lie algebra of formal vector fields in $n$-variables $x_{1}, \cdots, x_{n}$, preserving the volume form $d x_{1} \wedge \cdots \wedge d x_{n}$.

(3) $L_{s p}(2 n)$ : the Lie algebra of formal vector fields in $2 n$-variables $x_{1}, \cdots, x_{n}$, $y_{1}, \cdots, y_{n}$, preserving the symplectic form $\sum_{i=1}^{n} d x_{i} \wedge d y_{i}$.

(4) $L_{c t}(2 n+1):$ the Lie algebra of formal vector fields in $(2 n+1)$-variables $z, x_{1}, \cdots, x_{n}, y_{1}, \cdots, y_{n}$, preserving the contact form $d z+\frac{1}{2} \sum_{i=1}^{n} x_{i} d y_{i}-y_{i} d x_{i}$ up to functional factors.

We will not specify the ground field of each classical infinite Lie algebra. We have in mind it will always be the real or complex numbers. It is well-known that these classical infinite Lie algebras are "transitive" and algebraically simple [1]. In particular if the ground field is the field of complex numbers, four Lie algebras above mentioned are all of infinite transitive simple Lie algebras.

In the next place, we define the global classical infinite Lie algebras which are globally defined on differentiable manifolds. Here manifolds and other objects are assumed to be of class $C^{\infty}$.

$(1)^{\prime} \quad \mathscr{L}_{g l}(M):$ the Lie algebra of all vector fields defined on a manifold $M$.

Suppose that it is given a volume form $\eta$ on $M$.

$(2)^{\prime} \quad \mathscr{L}_{s l}(M)$ : the Lie algebra consisting of vector fields $X$ on $M$ satisfying $L(X) \eta=0$, where $L(X)$ denotes the Lie derivative with respect to $X$.

Suppose that $(M, \omega)$ is a symplectic manifold of $2 n$-dimension, that is, there is given a non-degenerate closed 2-form $\omega$ on $M$. 
$(3)^{\prime} \quad \mathscr{L}_{s p}(M, \omega):$ the Lie algebra consisting of vector fields $X$ on $M$ satisfying $L(X) \omega=0$.

Let $(M, \theta)$ be a $(2 n+1)$-dimensional contact manifold, that is, there is given a non-degenerate 1-form $\theta$ on $M$.

(4) $\mathscr{L}_{c t}(M, \theta):$ the Lie algebra consisting of vector fields $X$ on $M$ satisfying $L(X) \theta=f \theta$, where $f$ is some function depending on $X$.

Let $\mathscr{L}$ be a global classical infinite Lie algebra and $L$ be a formal classical infinite Lie algebra corresponding to $\mathscr{L}$. Then by definition, for any element $X$ of $\mathscr{L}$, the Taylor expansion of $X$ at $x \in M$ is an element of $L$. (Exactly speaking, we should write $L_{x}$ instead of $L$. But for any points $x$ and $y$ of $M, L_{x}$ is isomorphic to $L_{y}$ so that we will simply write $L$.) We call this algebra $L$ the formal algebra of $\mathscr{L}$ (at $x \in M$ ). For the precise definition of the formal algebra, see [8].

In $\S 2,3,4,5$ and 6 , we confine ourselves to the formal category, so we shall omit the adjective "formal".

\section{§ 2. Fundamental facts about the classical infinite Lie algebras}

In this section we briefly recall a few fundamental facts about the structure of the classical infinite Lie algebras. Details are referred to [1], [4] and [8].

Let $L$ be a classical infinite Lie algebra. If $L$ is one of $L_{g l}(n), L_{s l}(n)$ and $L_{s p}(2 n)$, then the natural filtration $\left\{L_{p}\right\}_{p \in Z}$ of $L$ is defined as follows:

$$
\begin{aligned}
& L_{p}=L \quad \text { for } p \leqq-1, \\
& L_{0}=\{X \in L ; \text { the value } X(0) \text { of } X \text { at the origin }=0\}, \\
& L_{p}=\left\{X \in L_{p-1} ;[X, L] \subset L_{p-1}\right\} \quad \text { for } p \geqq 1 .
\end{aligned}
$$

If $L$ is the contact Lie algebra $L_{c t}(2 n+1)$ (or simply $L_{c t}$ ), we define another filtration. It is defined inductively as follows:

$$
\begin{aligned}
L_{p} & =L_{c t} \quad \text { for } p \leqq-2, \\
L_{-1} & =\left\{X \in L_{c t} ;\langle X, \theta\rangle_{0}=0, \text { where } \theta \text { is the contact form }\right\}, \\
L_{0} & =\left\{X \in L_{c t} ; X(0)=0\right\}, \\
L_{p} & =\left\{X \in L_{p-1} ;\left[X, L_{-1}\right] \subset L_{p-1}\right\} \quad \text { for } p \geqq 1 .
\end{aligned}
$$

Since this filtration is compatible with usual one, we also denote by $\left\{L_{p}\right\}$ the filtration for the contact Lie algebra.

We topologize a classical infinite Lie algebra $L$ by assigning $\left\{L_{p}\right\}$ as a system of fundamental neighborhood of $L$. Then $L$ is a topological Lie algebra and it is separated and complete with respect to the filtration topology. We should remark that a Lie subalgebra $B$ of $L$ is closed if and only if $B=\bigcap_{p \in Z}\left(B+L_{p}\right)$.

Put $g_{p}(L)=L_{p} / L_{p+1}$. The graded Lie algebra gr $(L)$ associated with $L$ is defined by $\operatorname{gr}(L)=\sum_{p \in Z} \mathfrak{g}_{p}(L)$. For a Lie subalgebra $B$ of $L, \operatorname{gr}(B)$ is defined by $\operatorname{gr}(B)=$ $\sum_{p \in Z} \mathrm{~g}_{p}(B)$, where $\mathrm{g}_{p}(B)=B \cap L_{p} / B \cap L_{p+1}$. Since $\left[L_{p}, L_{q}\right] \subset L_{p+q}$, it holds that 
$\left[\mathfrak{g}_{p}(L), \mathfrak{g}_{q}(L)\right] \subset \mathfrak{g}_{p+q}(L)$ for all $p, q \in Z$. We denote by $\prod_{p \in Z} \mathfrak{g}_{p}(L)$ the topological completion of the graded Lie algebra $\sum_{p \in Z} \mathfrak{g}_{p}(L)$.

Remark. Let $L$ be a classical infinite Lie algebra. If $B$ is a closed (and hence complete) Lie subalgebra of $L$ with $\operatorname{gr}(B)=\operatorname{gr}(L)$, then $B$ coincides with $L$.

Let $L$ be one of $L_{g l}(n), L_{s l}(n)$ and $L_{s p}(2 n)$. Recall that $L=L_{-1}$ for these Lie algebras. Put $L_{-1} / L_{0}=V$. Then $V$ is a finite dimensional vector space. Let $V^{*}$ be the dual space of $V$ and $S^{p}\left(V^{*}\right)$ be the $p$-times symmetric tensor of $V^{*}$. Then $g_{p}(L)$ can be regarded as a subspace of $V \otimes S^{p+1}\left(V^{*}\right)$.

Proposition 2.1. Let $v$ be any non-zero vector of $V$. Then $\left[v, \mathfrak{g}_{p}(L)\right]=\mathfrak{g}_{p-1}(L)$ for all $p \geqq 0$.

proof. If $L=L_{\theta l}(n), \mathrm{g}_{p}(L)$ coincides with $V \otimes S^{p+1}\left(V^{*}\right)$. Hence the assertion clearly holds. Let $L=L_{s p}(2 n)$. In this case, $g_{p}(L)=s p(V)^{(p)}$, which is the $p$-th prolongation of the symplectic Lie algebra $s p(V)$. Let $\langle$,$\rangle be an anti-symmetric non-$ degenerate bilinear form on $V$. Then it induces an isomorphism of $V \rightarrow V^{*}$. And this isomorphism also induces an isomorphism $\psi_{p}: s p(V)^{(p)} \rightarrow S^{p+2}\left(V^{*}\right)$, which is defined by

$$
\psi_{p}(X)\left(v_{1}, \cdots, v_{p+2}\right)=\left\langle X\left(v_{1}, \cdots, v_{p+1}\right), v_{p+2}\right\rangle .
$$

Using these identifications, the bracket operation is naturally defined on $V^{*} \times S^{p+2}\left(V^{*}\right)$, which is induced from the Lie bracket operation on $V \times \operatorname{sp}(V)^{(p)}$. Let $v^{*}$ be the dual of $v \in V$ with respect to $\langle$,$\rangle . Now to prove the assertion, it$ has only to show that $\left[v^{*}, S^{p+2}\left(V^{*}\right)\right]=S^{p+1}\left(V^{*}\right)$. Let $\partial / \partial x_{1}, \cdots, \partial / \partial x_{n}, \partial / \partial y_{1}, \cdots$, $\partial / \partial y_{n}$ be a basis of $V$. Let $f \in S^{p+2}\left(V^{*}\right)$ be of the form $f=x_{1}^{\alpha_{1}} \cdots x_{n}^{\alpha_{n}} y_{1}^{\beta_{1}} \cdots y_{n}^{\beta_{n}}$, and $X$ be an element of $\operatorname{sp}(V)^{(p)}$ corresponding to $f$ through $\psi_{p}$. Then the above fact is easily obtained from the following equations:

$$
\begin{aligned}
& \psi_{p-1}\left(\left[\frac{\partial}{\partial x_{i}}, X\right]\right)= \begin{cases}x_{1}^{\alpha_{1}} \cdots x_{i}^{\alpha_{i}-1} \cdots x_{n}^{\alpha_{n}} y_{1}^{\beta_{1}} \cdots y_{n}^{\beta_{n}} & \left(\alpha_{i} \neq 0\right), \\
0 & \left(\alpha_{i}=0\right),\end{cases} \\
& \psi_{p-1}\left(\left[\frac{\partial}{\partial y_{i}}, X\right]\right\}= \begin{cases}x_{1}^{\alpha_{1}} \cdots x_{n}^{\alpha_{n}} y_{1}^{\beta_{1}} \cdots y_{i}^{\beta_{i}-1} \cdots y_{n}^{\beta_{n}} & \left(\beta_{i} \neq 0\right), \\
0 & \left(\beta_{i}=0\right) .\end{cases}
\end{aligned}
$$

Finally we consider the case of $L=L_{s l}(n)$. Let $v$ be a given non-zero vector. First note that $s l(V)$ is an involutive Lie algebra. For any $g \in G L(n)$, it holds that $g \cdot s l(V) \cdot g^{-1}=s l(V)$. Hence any basis of $V$ is generic, and it is also clear that $[v, s l(V)]=V$. Since the $p$-th prolongation $s l(V)^{(p)}$ of $s l(V)$ is involutive, we can conclude that any basis of $V$ is generic for $s l(V)^{(p)}$. Let $\left(v, e_{2}, \cdots, e_{n}\right)$ be a basis of $V$. Then $\left(v, e_{2}, \cdots, e_{n}\right)$ is a generic basis for $\operatorname{sl}(V)^{(p)}$. Therefore, by definition, $T_{v}: s l(V)^{(p)} \rightarrow s l(V)^{(p-1)}$ is surjective for $p>0$, where $T_{v}$ is a linear mapping defined by $T_{v}(X)=[v, X]$ for $X \in \operatorname{sl}(V)^{(p)}$.

q.e.d.

In the rest of this section, we will state some fundamental properties of the 
contact Lie algebra. Details are referred to Morimoto-Tanaka [4]. We will denote the graded Lie algebra associated with $L_{c t}(2 n+1)$ by $\sum_{p \geqq-2} c_{p}$. (Recall that in the case of $L_{c t}(2 n+1)$ we have adopted a new filtration.) In $\S 6$ we will also use this notation.

Proposition 2.2 (see [4]). (i) $c_{-2}$ is one dimensional. Let $u$ be a basis of $c_{-2}$. Then ad $(u)$ maps $c_{p}$ onto $c_{p-2}$ for all $p \geqq 0$.

(ii) $\left[c_{-1}, c_{-1}\right]=c_{-2}$.

(iii) $c_{-1}$ is $2 n$-dimensional. Let $s p\left(c_{-1}\right)$ denote the symplectic Lie algebra over $c_{-1}$ and $s p\left(c_{-1}\right)^{(p)}$ be the $p$-th prolongation of $s p\left(c_{-1}\right)$. Then for $p \geqq 0$, there exists an $s p\left(c_{-1}\right)$-invariant subspace ${ }_{z_{p}}$ of $c_{p}$ and $c_{p}$ is decomposed into

$$
c_{p}=s p\left(c_{-1}\right)^{(p)}+z_{p} \quad \text { (direct sum) }
$$

\section{§3. Proof of the main theorem (the case of $L_{g l}(n)$ )}

Throughout this section $L$ denotes $L_{g l}(n)$. In this case the proof is done by the analogous method as that of K. Masuda [2]. Let $B$ be a proper Lie subalgebra of $L$ with $\operatorname{codim} B=c<\infty$. We define an ideal $B^{\prime}$ of $B$ by $B^{\prime}=\{X \in B ;[X, L] \subset B\}$. Then for any $X \in B$, a linear mapping $T(X): L / B \rightarrow L / B$ is induced from the adjoint mapping. Since $B^{\prime}$ is the kernel of the mapping $X \rightarrow T(X)$ of $B$ into the space of endomorphisms of $L / B$, we have $\operatorname{dim} L / B^{\prime} \leqq c^{2}+c$. Let $F$ denote the ring of formal power series of $n$-variables. Let $g \in F$ be a homogeneous polynomial of degree one, and $Z$ be a vector field of $L$ satisfying $Z(g)=1$. We define a subspace $\mathscr{P}^{\prime}$ of the polynomial ring $\mathscr{P}$ of one variable by

$$
\mathscr{P}^{\prime}=\left\{P \in \mathscr{P} ; g P(g) Z, P(g) Z \in B^{\prime}\right\} .
$$

Since $\operatorname{dim} \mathscr{P} / \mathscr{P}^{\prime} \leqq 2\left(c^{2}+c\right)$, we can find a non-trivial polynomial $P \in \mathscr{P}^{\prime}$ with $\operatorname{deg} P$ $\leqq m$, where $m=2\left(c^{2}+c\right)$. Then we have for any $X \in L$

$$
B \ni[P(g) Z, g X]-[g P(g) Z, X]=P(g) X+X(g) P(g) Z .
$$

Substituting $X(g) Z$ for $X$, we have $P(g) X \in B$ and hence $P(g) L \subset B$. Put $P(g)=$ $g^{p}\left(a_{0}+a_{1} g+\cdots+a_{q} g^{q}\right),\left(a_{0} \neq 0\right)$. Then it holds that $g^{p} L \subset B$. Combining this with $g^{m-p} L \subset L$, we obtain $g^{m} L \subset B$. Since $g$ is an arbitrary homogeneous polynomial of degree one, we can easily conclude that $S^{m}\left(V^{*}\right) L=L_{m-1} \subset B$. Hence we see that $B$ is a closed Lie subalgebra of $L$. Combining the remark of the previous section with Proposition 2.1, B must be contained in $L_{0}$. Thus we have proved

Theorem 3.1. Let $B$ be a proper Lie subalgebra of finite codimension of $L=L_{g l}(n)$. Then there exists a positive integer $k$ such that $L_{k} \subset B \subset L_{0}$.

\section{§4. Proof of a lemma (the hamiltonian case)}

To prove the theorem for $L_{s p}(2 n)$, we can not follow the method of $L_{g l}(n)$. So 
we begin with defining the "Poisson algebra". Let $V$ be a $2 n$-dimensional vector space with a basis $\partial / \partial x_{1}, \cdots, \partial / \partial x_{n}, \partial / \partial y_{1}, \cdots, \partial / \partial y_{n}$. Let $V^{*}$ be the dual of $V$ and $S^{p}\left(V^{*}\right)$ be the $p$-times symmetric tensor of $V^{*}$. The ring of formal power series, which is denoted by $F(V)$, is the complete deirect sum $\prod_{p=0}^{\infty} S^{p}\left(V^{*}\right)$. For any $f, g \in F(V)$, the Poisson bracket is defined by

$$
\{f, g\}=\sum_{i=1}^{n} \frac{\partial f}{\partial x_{i}} \frac{\partial g}{\partial y_{i}}-\frac{\partial f}{\partial y_{i}}-\frac{\partial g}{\partial x_{i}} .
$$

Then we see that $F(V)$ is a Lie algebra with respect to this bracket product. This Lie algebra $F(V)$ is called the Poisson algebra.

Let $\omega=\sum_{i=1}^{n} d x_{i} \wedge d y_{i}$. Then $L_{s p}(2 n)$ is defined by

$$
L_{s p}(2 n)=\left\{X \in L_{o l}(2 n) ; L(X) \omega=0\right\} .
$$

We define a linear mapping $\varphi$ of $F(V)$ to $L_{s p}(2 n)$ by

$$
\varphi(f)=\sum_{i=1}^{n} \frac{\partial f}{\partial x_{i}} \frac{\partial}{\partial y_{i}}-\frac{\partial f}{\partial y_{i}} \frac{\partial}{\partial x_{i}} .
$$

Then it is well-known that $\varphi$ gives an onto Lie homomorphism (cf. [7]). From now on we simply write $L$ for $L_{s p}(2 n)$ and $F$ for $F(V)$. Put $F_{p}=\prod_{k \geqq p+2} S^{k}\left(V^{*}\right)$. Then $\varphi\left(F_{p}\right)=L_{p}$. Since $\left\{F_{p}, F_{q}\right\} \subset F_{p+q}$, each $F_{p}(p \geqq 0)$ gives a typical example of a Lie subalgebra of finite codimension of $F$.

In order to prove our main theorem in $\S 7$, the following lemma is essential.

Lemma 4.1. Let $G$ be a proper Lie subalgebra of $F$ with $\operatorname{dim} F / G=c<\infty$. Then $G$ contains $x_{1}^{m} F, \cdots, x_{n}^{m} F, y_{1}^{m} F, \cdots, y_{n}^{m} F$, where $m=2\left(c^{2}+c\right)$. Furthermore setting $N=2 n(m-1)+1, G$ contains $F_{N-2}$.

Proof. Put $G^{\prime}=\{f \in G ;\{f, F\} \subset G\} . \quad$ Then $G^{\prime}$ is an ideal of $G$ with $\operatorname{dim} F / G^{\prime}$ $\leqq c^{2}+c$. Let $\mathscr{P}$ be the polynomial ring of one variable. Define a subspace $\mathscr{P}^{\prime}$ of $\mathscr{P}$ for $i$ fixed by

$$
\mathscr{P}^{\prime}=\left\{P \in \mathscr{P} ; x_{i} P\left(x_{i}\right) y_{i}, P\left(x_{i}\right) y_{i} \in G^{\prime}\right\} .
$$

By the same way as the previous section, we can find a non-trivial polynomial $P \in \mathscr{P}^{\prime}$ with $\operatorname{deg} P \leqq 2\left(c^{2}+c\right)=m$ since $\operatorname{dim} \mathscr{P} / \mathscr{P}^{\prime} \leqq m$. Fix this $P \in \mathscr{P}^{\prime}$. For any $f \in F$ we have

$$
\begin{aligned}
& G \ni\left\{x_{i} f, P\left(x_{i}\right) y_{i}\right\}=x_{i}\left\{f, P\left(x_{i}\right) y_{i}\right\}+P\left(x_{i}\right) f, \\
& G \ni\left\{f, x_{i} P\left(x_{i}\right) y_{i}\right\}=x_{i}\left\{f, P\left(x_{i}\right) y_{i}\right\}-P\left(x_{i}\right) \frac{\partial f}{\partial y_{i}} y_{i},
\end{aligned}
$$

and hence $\left.G \ni P\left(x_{i}\right)\left(f+\left(\partial f / \partial y_{i}\right) y_{i}\right)\right)$. Since a mapping $f \rightarrow f+\left(\partial f / \partial y_{i}\right) y_{i}$ maps $F$ onto itself, we have $P\left(x_{i}\right) F \subset G$. Put $P\left(x_{i}\right)=x_{i}^{r}\left(a_{0}+a_{1} x_{i}+\cdots+a_{s} x_{i}^{s}\right),\left(a_{0} \neq 0\right)$ and $h\left(x_{i}\right)=a_{0}+\cdots+a_{s} x_{i}^{s}$. Then $h\left(x_{i}\right)^{-1} \in F$, and for any $f \in F, G \ni P\left(x_{i}\right) h\left(x_{i}\right)^{-1} f=x_{i}^{r} f$. 
Combining this with $x_{i}^{m-r} F \subset F$, we have $x_{i}^{m} F \subset G$. Similarly we also have $y_{i}^{m} F \subset G$. (Note that $m$ does not depend on $i$.)

In the next place, we will prove the latter half. Put $N=2 n(m-1)+1$. In the following expression,

$$
\left(\sum_{i=1}^{n} a_{i} x_{i}+b_{i} y_{i}\right)^{N}=\sum_{|\alpha|+|\beta|=N} C_{\alpha \beta} x_{1}^{\alpha_{1}} \cdots x_{n}^{\alpha_{n}} y_{1}^{\beta_{1}} \cdots y_{n}^{\beta_{n}},
$$

where $\alpha=\left(\alpha_{1}, \cdots, \alpha_{n}\right), \beta=\left(\beta_{1}, \cdots, \beta_{n}\right)$ and $|\alpha|=\sum_{i=1}^{n} \alpha_{i},|\beta|=\sum_{i=1}^{n} \beta_{i}$, at least one of these $\alpha_{1}, \cdots, \alpha_{n}, \beta_{1}, \cdots, \beta_{n}$ is greater than or equal to $m$. This implies $\left(\sum_{i=1}^{n} a_{i} x_{i}\right.$ $\left.+b_{i} y_{i}\right)^{N} \cdot F \subset G$. Since $2 n$ constants $a_{1}, \cdots, a_{n}, b_{1}, \cdots, b_{n}$ are arbitrary, we have $G \supset S^{N}\left(V^{*}\right) F=F_{N-2}$. This completes the proof. $\quad$ q.e.d.

\section{$\S 5$. Proof of a lemma (the case of $L_{s l}(n)$ )}

In this section, we prove an important lemma in the case of $L_{s l}(n),(n \geqq 3)$. Let $V$ be an $n$-dimensional vector space with a basis $\partial / \partial x_{1}, \cdots, \partial / \partial x_{n}$. We denote by $F$ the ring of formal power series with $n$-variables $x_{1}, \cdots, x_{n}$. Let $\Lambda^{n-2}$ (resp. $C^{n-1}$ ) be the space of (formal) ( $n-2)$-forms (resp. closed (n-1)-forms). In this case, the exterior derivative $d: \Lambda^{n-2} \rightarrow C^{n-1}$ is surjective. For the volume form $\eta=d x_{1} \wedge \cdots$ $\wedge d x_{n}, L_{s l}(n)$ is defined by $L_{s l}(n)=\left\{X \in L_{o l}(n) ; L(x) \eta=0\right\}$. There is a canonical isomorphism between $L_{s l}(n)$ and $C^{n-1}$. The isomorphism is given by $X \rightarrow i(X) \eta$ for $X \in L_{s l}(n)$, where $i(X)$ denotes the interior product by $X$. Let $\tau$ be the inverse mapping of this isomorphism. Put $\#=\tau \circ d$. Then $\#: A^{n-2} \rightarrow L_{s l}(n)$ is surjective. Let $\gamma_{i j} \in \Lambda^{n-2}$ be of the form

$$
\gamma_{i j}=(-1)^{i+j+1} d x_{1} \wedge \cdots \wedge \widehat{d x_{i}} \wedge \cdots \wedge \widehat{d x_{j}} \wedge \cdots \wedge d x_{n}
$$

Then by a simple calculation, we see that

$$
\left[\left(f \gamma_{i j}\right)^{\#},\left(g \gamma_{i j}\right)^{\#}\right]=-\left(\{f, g\}_{i j} \gamma_{i j}\right)^{\#} \quad \text { for any } f, g \in F,
$$

where $\{f, g\}_{i j}$ is the Poisson bracket of $f$ and $g$ with respect to $x_{i}$ and $x_{j}$, that is,

$$
\{f, g\}_{i j}=\frac{\partial f}{\partial x_{i}} \frac{\partial g}{\partial x_{j}}-\frac{\partial g}{\partial x_{i}} \frac{\partial f}{\partial x_{j}} .
$$

Any $\gamma \in \Lambda^{n-2}$ is written as $\gamma=\sum_{i<j} f_{i j} \gamma_{i j}\left(f_{i j} \in F\right)$. We define a subspace $\Lambda_{N}^{n-2}$ of $\Lambda^{n-2}$ by $\Lambda_{N}^{n-2}=\left\{\gamma \in \Lambda^{n-2}\right.$; each $\left.f_{i j} \in \prod_{p \geqq N} S^{p}\left(V^{*}\right)\right\}$.

From now on we will simply write $L$ for $L_{s l}(n)$.

Lemma 5.1. Let $B$ be a proper Lie subalgebra of $L$ with $\operatorname{dim} L / B=c<\infty$. Then $B \supset\left(\Lambda_{N}^{n-2}\right)^{\#}$ where $N=n\left\{2\left(c^{2}+c\right)-1\right\}+1$.

Proof. As in the case of $L_{s p}(2 n)$, define an ideal $B^{\prime}$ of $B$ by $B^{\prime}=\{X \in B ;[X, L]$ $\subset B\}$. Fix a pair $i, j(i<j)$ arbitrarily among $1, \cdots, n$. Let $\mathscr{P}$ be the polynomial ring of one variable. Define a subspace $\mathscr{P}_{i j}^{\prime}$ of $\mathscr{P}$ by 


$$
\mathscr{P}_{i j}^{\prime}=\left\{P \in \mathscr{P} ;\left(x_{i} P\left(x_{i}\right) x_{j} \gamma_{i j}\right)^{\#},\left(P\left(x_{i}\right) x_{j} \gamma_{i j}\right)^{\#} \in B^{\prime}\right\} .
$$

Since $\operatorname{dim} \mathscr{P} / \mathscr{P}_{i j}^{\prime} \leqq 2\left(c^{2}+c\right)$, there exists a non-trivial polynomial $P \in \mathscr{P}_{i \text { j }}^{\prime}$ such that $\operatorname{deg} P \leqq 2\left(c^{2}+c\right)$. Put $m=2\left(c^{2}+c\right)$. Then by using (5.1) and (5.2) we have for any $f \in F$,

$$
\begin{aligned}
& B \ni\left[\left(x_{i} f \gamma_{i j}\right)^{\#},\left(P\left(x_{i}\right) x_{j} \gamma_{i j}\right)^{\sharp}\right]=-\left(x_{i}\left\{f, P\left(x_{i}\right) x_{j}\right\}_{i j} \gamma_{i j}\right)^{\#}-\left(P\left(x_{i}\right) f \gamma_{i j}\right)^{\#}, \\
& B \ni\left[\left(f \gamma_{i j}\right)^{\#},\left(x_{i} P\left(x_{i}\right) x_{j} \gamma_{i j}\right)^{\sharp}\right]=-\left(x_{i}\left\{f, P\left(x_{i}\right) x_{j}\right\}_{i j} \gamma_{i j}\right)^{\#}-\left(P\left(x_{i}\right) x_{j}\left\{f, x_{i}\right\}_{i j} \gamma_{i j}\right)^{\#},
\end{aligned}
$$

and hence $B \ni\left(P\left(x_{i}\right)\left(f+\left(\partial f / \partial x_{j}\right) x_{j}\right) \gamma_{i j}\right)^{\#}$.

By the same argument as the hamiltonian case, we can conclude that $B \supset$ $\left(x_{i}^{m} \cdot F \cdot \gamma_{i j}\right)^{\#}$. Substituting $x_{j}$ for $x_{i}$ in (5.3) and (5.4), we also have $B \supset\left(x_{j}^{m} \cdot F \cdot \gamma_{i j}\right)^{\#}$.

If $k \neq i, k \neq j$, we define a subspace $\mathscr{P}_{k}^{\prime}$ by

$$
\mathscr{P}_{k}^{\prime}=\left\{P \in \mathscr{P} ;\left(P\left(x_{k}\right) x_{j} \gamma_{i j}\right)^{\#} \in B^{\prime}\right\} .
$$

Then $\operatorname{dim} \mathscr{P} \mid \mathscr{P}_{k}^{\prime} \leqq \frac{1}{2} m$ and we can find a non-trivial polynomial $P \in \mathscr{P}_{k}^{\prime}$ with $\operatorname{deg} P \leqq \frac{1}{2} m$. For any $f \in F$ we have

$$
\begin{aligned}
& B \ni\left[\left(x_{i} f \gamma_{i j}\right)^{\#},\left(P\left(x_{k}\right) x_{j} \gamma_{i j}\right)^{\sharp}\right] \\
& \left.\quad=-\left(x_{i} f, P\left(x_{k}\right) x_{j}\right\}_{i j} \gamma_{i j}\right)^{\#}=-\left(P\left(x_{k}\right)\left(f+\frac{\partial f}{\partial x_{i}} x_{i}\right) \gamma_{i j}\right)^{\#} .
\end{aligned}
$$

This implies $\left(\left(x_{k}\right)^{m / 2} \cdot F \cdot \gamma_{i j}\right)^{\sharp} \subset B$ and hence $\left(x_{k}^{m} \cdot F \cdot \gamma_{i j}\right)^{\sharp} \subset B$. Thus it holds that $B \supset\left(x_{l}^{m} \cdot F \cdot \gamma_{i j}\right)^{\sharp}$ for $l=1, \cdots, n$, and hence $B \supset\left(\prod_{p \geqq N} S^{p}\left(V^{*}\right) \gamma_{i j}\right)^{\#}$, where $N=n(m-$ $1)+1$. This fact is also true for each pair $i, j(i<j)$ and we complete the proof.

q.e.d.

\section{§6. Proof of lemmata (the contact case)}

Let $V$ be a $(2 n+1)$-dimensional vector space with a basis $\partial / \partial z, \partial / \partial x_{1}, \cdots, \partial / \partial x_{n}$, $\partial / \partial y_{1}, \cdots, \partial / \partial y_{n}$, and $F$ be the ring of formal power series of $(2 n+1)$-variables $z, x_{1}, \cdots, x_{n}, y_{1}, \cdots, y_{n}$. For the contact form $\theta=d z+\frac{1}{2} \sum_{i=1}^{n} x_{i} d y_{i}-y_{i} d x_{i}$, the contact Lie algebra $L=L_{c t}(2 n+1)$ is defined by $L=\left\{X \in L_{g l}(2 n+1) ; L(X) \theta=u \theta, u\right.$ is a function of $F$ depending on $X$ \}. We first define the "Jacobi bracket" on $F$. Since $\theta$ is non-degenerate, it induces a linear isomorphism of $L$ to $F$ defined by $X \rightarrow i(X) \theta$. Let $K$ denote the inverse mapping of this isomorphism. Then by a simple calculation, we have

$$
\begin{aligned}
K(f)= & \left\{f-\frac{1}{2} \sum_{i=1}^{n}\left(x_{i} \frac{\partial f}{\partial x_{i}}+y_{i} \frac{\partial f}{\partial y_{i}}\right)\right\} \frac{\partial}{\partial z}-\sum_{i=1}^{n}\left(\frac{\partial f}{\partial y_{i}}-\frac{1}{2} x_{i} \frac{\partial f}{\partial z}\right) \frac{\partial}{\partial x_{i}} \\
& +\sum_{i=1}^{n}\left(\frac{\partial f}{\partial x_{i}}+\frac{1}{2} y_{i} \frac{\partial f}{\partial z}\right) \frac{\partial}{\partial y_{i}}, \quad \text { for any } f \in F .
\end{aligned}
$$

Using (6.1), $K$ induces a Lie algebra structure on $F$, of which bracket operation 
is called the Jacobi bracket and will be denoted by $\{$,$\} . Then it has the following$ expression:

$$
\begin{aligned}
\{f, g\}= & \sum_{i=1}^{n}\left(-\frac{\partial f}{\partial x_{i}} \frac{\partial g}{\partial y_{i}}-\frac{\partial f}{\partial y_{i}} \frac{\partial g}{\partial x_{i}}\right)+\left\{f-\frac{1}{2} \sum_{i=1}^{n}\left(x_{i} \frac{\partial f}{\partial x_{i}}+y_{i} \frac{\partial f}{\partial y_{i}}\right)\right\} \frac{\partial g}{\partial z} \\
& -\left\{g-\frac{1}{2} \sum_{i=1}^{n}\left(x_{i} \frac{\partial g}{\partial x_{i}}+y_{i} \frac{\partial g}{\partial y_{i}}\right)\right\} \frac{\partial f}{\partial z} .
\end{aligned}
$$

Now we introduce a filtration $\left\{F_{p}\right\}_{p \in Z}$ of $F$, which was first introduced by $\mathrm{T}$. Morimoto [3]. In the first place we define ord $(f)$ for any $f \in F$. For a monomial $x^{\alpha} y^{\beta} z^{\gamma}$, where $\alpha=\left(\alpha_{1}, \cdots, \alpha_{n}\right), \beta=\left(\beta_{1}, \cdots, \beta_{n}\right)$ and $x^{\alpha}=x_{1}^{\alpha_{1}} \cdots x_{n}^{\alpha_{n}}$ and $y^{\beta}=y_{1}^{\beta_{1}} \cdots y_{n}^{\beta_{n}}$, ord $\left(x^{\alpha} y^{\beta} z^{\gamma}\right)$ is defined as

$$
\text { ord }\left(x^{\alpha} y^{\beta} z^{r}\right)=|\alpha|+|\beta|+2 \gamma-2
$$

where $|\alpha|=\sum_{i=1}^{n} \alpha_{i},|\beta|=\sum_{i=1}^{n} \beta_{i}$. For any $f \in F$, ord $(f)$ is defined to be the minimum of orders of non-zero monomials. Then we define a filtration $\left\{F_{p}\right\}$ of $F$ by

$$
F_{p}=\{f \in F \text {; ord }(f) \geqq p\}, \quad \text { for } p \in Z .
$$

This is the filtration which is compatible with that of $L$, that is, we see that $K\left(F_{p}\right)=L_{p}$.

From now on we regard $F$ as a Lie algebra with respect to the Jacobi bracket. We denote by $\tilde{F}$ the Lie subalgebra of $F$, which is consisting of $f \in F$ such that $\partial f / \partial z=0$. Namely $\tilde{F}$ is the ring of formal power series of $2 n$-varialbes $x_{1}, \cdots, x_{n}$, $y_{1}, \cdots, y_{n}$. Let $W^{*}$ be a subspace of $V^{*}$ with a basis $x_{1}, \cdots, x_{n}, y_{1}, \cdots, y_{n}$. Put $\widetilde{F}^{N}=\prod_{p \geqq N} S^{p}\left(W^{*}\right)$.

Lemma 6.1. Let $G$ be a proper Lie subalgebra of $F$ with $\operatorname{dim} F / G=c<\infty$. Then $G \supset z^{N} \cdot F, \tilde{F}^{N}$ where $m=2\left(c^{2}+c\right)$ and $N=2 n(m-1)+1$. Moreover there exists $a$ positive integer $k$ such that $G \supset F_{k}$.

Proof. First we will show that $z^{N} \cdot F \subset G$. Let $\mathscr{P}^{\prime}$ be a subspace of the polynomial ring of one variable $\mathscr{P}$, which is defined by

$$
\mathscr{P}^{\prime}=\left\{P \in \mathscr{P} ; x_{i} P(z), y_{i} P(z) \in G^{\prime}\right\},
$$

where $G^{\prime}$ is an ideal of $G$ defined by $G^{\prime}=\{f \in G ;\{f, F\} \subset G\}$, and $i$ is arbitrarily fixed. Put $2\left(c^{2}+c\right)=m$. Then there exists a non-trivial polynomial $P \in \mathscr{P}^{\prime}$ with $\operatorname{deg} P \leqq m$. For any $f \in F$ we have

$$
\begin{gathered}
G \ni\left\{x_{i} P(z), y_{i} f\right\}=P(z) f+P(z) y_{i} \frac{\partial f}{\partial y_{i}}+\frac{1}{2} x_{i} y_{i} P(z) \frac{\partial f}{\partial z} \\
\quad-\frac{1}{2} x_{i} y_{i} P^{\prime}(z) f+\frac{1}{2} x_{i}^{2} y_{i} P^{\prime}(z) \frac{\partial f}{\partial x_{i}}+\frac{1}{2} x_{i} y_{i}^{2} P^{\prime}(z) \frac{\partial f}{\partial y_{i}} \\
G \ni\left\{y_{i} P(z), x_{i} f\right\}=-P(z) f-P(z) x_{i}-\frac{\partial f}{\partial x_{i}}+\frac{1}{2} x_{i} y_{i} P(z) \frac{\partial f}{\partial z}
\end{gathered}
$$




$$
-\frac{1}{2} x_{i} y_{i} P^{\prime}(z) f+\frac{1}{2} x_{i}^{2} y_{i} P^{\prime}(z) \frac{\partial f}{\partial x_{i}}+\frac{1}{2} x_{i} y_{i}^{2} P^{\prime}(z) \frac{\partial f}{\partial y_{i}},
$$

and hence $G \ni P(z)\left(2 f+x_{i}\left(\partial f / \partial x_{i}\right)+y_{i}\left(\partial f / \partial y_{i}\right)\right)$.

Since a mapping $f \rightarrow 2 f+x_{i}\left(\partial f / \partial x_{i}\right)+y_{i}\left(\partial f / \partial y_{i}\right)$ maps $F$ onto itself, we have $P(z) F \subset G$, and this implies $z^{m} \cdot F \subset G$.

In the next place, we will prove that $G \supset \widetilde{F}^{N}$. Note that the restriction of the Jacobi bracket to $\tilde{F}$ is just the Poisson bracket on $\tilde{F}$. Set $\tilde{G}=G \cap \tilde{F}$. Since $\tilde{F} / \tilde{G}$ is isomorphic to $(\tilde{F}+G) / G$ as linear spaces, we have

$$
c^{\prime}=\operatorname{dim} \tilde{F} / \tilde{G}=\operatorname{dim}(\tilde{F}+G) / G \leqq \operatorname{dim} F / G=c .
$$

Now $\tilde{G}$ is a Lie aubalgebra of finite codimension of $\tilde{F}$ (with respect to the Poisson bracket). Applying Lemma 4.1 to this case, we see that, $\widetilde{G} \supset \widetilde{F}_{N^{\prime}-2}=\widetilde{F}^{N^{\prime}}$, where $N^{\prime}=2 n\left(m^{\prime}-1\right)+1$ and $m^{\prime}=2\left(\left(c^{\prime}\right)^{2}+c^{\prime}\right)$. Thus we have proved $G \supset \tilde{F}^{N}, z^{N} \cdot F$, where $N=2 n(m-1)+1$.

Finally using these results we will show that there exists a positive integer $k$ such that $G \supset F_{k}$. Let $g=\prod_{|p|+|q| \geqq N} A_{p q} x^{p} y^{q}$ be any element of $\widetilde{F}^{N}$ where $p=\left(p_{1}\right.$, $\left.\cdots, p_{n}\right), \quad q=\left(q_{1}, \cdots, q_{n}\right)$. Put $\left.h=\prod_{|p|+|q| \geq N}\left\{A_{p q} / N(1-(|p|+|q|) / 2)\right)\right\} x^{p} y^{q} \in \widetilde{F}^{N}$. Then $G \ni\left\{h, z^{N}\right\}=z^{N-1} g$. Hence we have $z^{N-1} \widetilde{F}^{N} \subset G$. Similarly we obtain $z^{N-2} \widetilde{F}^{2 N}$, $z^{N-3} \widetilde{F}^{3 N}, \cdots, z \widetilde{F}^{N(N-1)} \subset G$. Let $\left\{x^{\alpha} y^{\beta} z^{r}\right\},\left(|\alpha|+|\beta|+\gamma=N^{2}-1\right)$ be a basis of $S^{N^{2}-1}\left(V^{*}\right)$. For a monomial $x^{\alpha} y^{\beta} z^{\gamma} \in S^{N^{2}-1}\left(V^{*}\right)$ and for any $f \in F, x^{\alpha} y^{\beta} z^{\gamma} f$ is clearly contained in $G$ if $\gamma \geqq N$. (Recall that $z^{N} F \subset G$.) If $\gamma \leqq N-1$, put $f=\prod_{p, q, r} B_{p q r} x^{p} y^{q} z^{r}$ and expand $x^{\alpha} y^{\beta} z^{\gamma} f$ as

$$
\begin{aligned}
x^{\alpha} y^{\beta} z^{\gamma} f= & z^{\gamma}\left(\prod_{p, q} B_{p q 0} x^{p+\alpha} y^{q+\beta}\right)+z^{\gamma+1}\left(\prod_{p, q} B_{p q 1} x^{p+\alpha} y^{q+\beta}\right) \\
& +\cdots+z^{N-1}\left(\prod_{p, q} B_{p q(N-1)} x^{p+\alpha} y^{q+\beta}\right)+A,
\end{aligned}
$$

where $A \in z^{N} F$. Since $|\alpha|+|\beta|=N^{2}-1-\gamma \geqq N^{2}-N$, we also have $x^{\alpha} y^{\beta} z^{r} f \in G$. This means that $S^{N^{2}-1}\left(V^{*}\right) F \subset G$. Put $k=2\left(N^{2}-2\right)$. Then $|p|+|q|+2 r-2 \geqq k$ implies $|p|+|q|+r \geqq N^{2}-1$, and hence $G \supset F_{k}$.

q.e.d.

Lemma 6.2. Let $B$ be a "closed" proper Lie subalgebra of finite codimension of $L=L_{c t}(2 n+1)$. Then $B$ is contained in $L_{0}$ with respect to the filtration defined in $\S 2$.

Proof. Let $\operatorname{gr}(L)=c_{-2}+c_{-1}+\cdots+c_{p}+c_{p+1}+\cdots$. By the assumption of $B, \operatorname{gr}(B)$ is written as $\operatorname{gr}(B)=c_{-2}^{\prime}+c_{-1}^{\prime}+\cdots+c_{p}^{\prime}+c_{p+1}+\cdots$. Assume that $c_{-2}^{\prime} \neq 0$. (Since $c_{-2}$ is one-dimensional, this means $c_{-2}^{\prime}=c_{-2}$.) Then by Proposition 2.2 (i), we have $\operatorname{gr}(B)=\operatorname{gr}(L)$. Since $B$ is closed, this implies $B=L$. Thus we must have $c_{-2}^{\prime}=0$. Using this fact and Proposition 2.2 (ii), we also see that $c_{-1}^{\prime}$ is a proper subspace of $c_{-1}$. Next assume that there exists a non-zero vector $v$ in $c_{-1}^{\prime}$. Then combining $\left[v, s p\left(c_{-1}\right)^{(p+1)}\right]=s p\left(c_{-1}\right)^{(p)}$ with Proposition 2.2 (iii), we have

$$
c_{p}^{\prime} \supset\left[v, c_{p+1}\right] \supset s p\left(c_{-1}\right)^{(p)},
$$


and hence $c_{p-1}^{\prime} \supset\left[v, c_{p}^{\prime}\right] \supset\left[v, s p\left(c_{-1}\right)^{(p)}\right]=s p\left(c_{-1}\right)^{(p-1)}$. Repeating this procedure, we get $c_{0}^{\prime} \supset s p\left(c_{-1}\right)$. This implies

$$
c_{-1}^{\prime} \supset\left[c_{0}^{\prime}, c_{-1}^{\prime}\right] \supset\left[s p\left(c_{-1}\right), c_{-1}^{\prime}\right] .
$$

Since $s p\left(c_{-1}\right)$ irreducibly acts on $c_{-1}, c_{-1}^{\prime}$ should coincide with $c_{-1}$. This is a contradiction. Thus we have proved that $c_{-2}^{\prime}=c_{-1}^{\prime}=0$. Clearly this implies $B \subset L_{0}$.

q.e.d.

\section{§7. Proof of the main theorem (the cases of $L_{s p}(2 n), L_{s l}(n)$ and $L_{c t}(2 n+1)$ )}

In $\S 4,5$ and 6 , we proved some important lemmata. In this section, we will carry out the proof of the main theorem by using them.

Theorem 7.1. Let $L$ denote one of the Lie algebras $L_{s p}(2 n), L_{s l}(n)$ and $L_{c t}(2 n+1)$. Let $B$ be a proper Lie subalgebra of $L$ with $\operatorname{dim} L / B=c<\infty$. Then there exists $a$ positive integer $k$ such that $L_{k} \subset B \subset L_{0}$.

Proof. If it can be shown that $B$ contains $L_{k}$ for some positive integer $k$, then using a remark in $\S 2$ with Proposition 2.1 for $L_{s p}(2 n)$ and $L_{s l}(n)$, and with Lemma 6.2 for $L_{c t}(2 n+1)$, we can conclude that $B$ is contained in $L_{0}$. So we have only to show that $B$ contains $L_{k}$.

1) The case $L=L_{s p}(2 n)$ : Let $F$ be the Poisson algebra, and $\varphi$ be the Lie homomorphism of $F$ onto $L$ defined in $\S 4$. Put $\varphi^{-1}(B)=G$. Then $G$ is a proper Lie subalgebra of $F$ with $\operatorname{dim} F / G=c$. Hence by Lemma 4.1, $G \supset F_{N-2}$ and this implies $B \supset L_{k}$, where $k=N-2$.

2) The case $L=L_{s l}(n)$ : Note that $d\left(\Lambda_{N}^{n-2}\right)$ is equal to $C_{N-1}^{n-1}=C^{n-1} \cap \Lambda_{N-1}$, and recall that $L_{N-2}$ is isomorphic to $C_{N-1}^{n-1}$. Then by Lemma 5.1 , the assertion is clearly obtained.

3) The case $L=L_{c t}(2 n+1)$ : Let $K$ be the Lie isomorphism of $F$ to $L$ defined by (6.1). Then $K^{-1}(B)$ is a Lie subalgebra of codimension $c$ of $F$. By Lemma 6.1, there exists a positive integer $k$ such that $K^{-1}(B) \supset F_{k}$. Thus $B \supset L_{k}$.

q.e.d.

In the next place, we will prove another theorem from the global version. Let $\mathscr{L}$ be a global classical infinite Lie algebra on a differentiable manifold $M$. It is well-known that for each of a volume form $\eta$, a symplectic form $\omega$, and a contact form $\theta$, there exist a coordinate neighborhood and a coordinate system such that it is expressed in the following form:

$$
\begin{aligned}
& \eta=d x_{1} \wedge d x_{2} \wedge \cdots \wedge d x_{n}, \\
& \omega=\sum_{i=1}^{n} d x_{i} \wedge d y_{i}, \\
& \theta=d z+\frac{1}{2} \sum_{i=1}^{n} x_{i} d y_{i}-y_{i} d x_{i} .
\end{aligned}
$$


We denote the above standard coordinate neighborhood (resp. coordinate system) by $\mathscr{L}$-coordinate neighborhood (resp. $\mathscr{L}$-coordinate system). The corresponding formal classical infinite Lie algebras of $\mathscr{L}_{g l}(M), \mathscr{L}_{s p}(M, \omega), \mathscr{L}_{s l}(M, \eta)$ and $\mathscr{L}_{c t}(M, \theta)$ are respectively $L_{g l}(n), L_{s p}(2 n), L_{s l}(n)$ and $L_{c t}(2 n+1)$.

Theorem 7.2. $\quad$ Let $\mathscr{B}$ be a proper Lie subalgebra of finite codimension of a global classicla infinite Lie algebra $\mathscr{L}$. Then it holds either a) or b).

a) There exist a finite number of points $a_{1}, \cdots, a_{r}$ of $M$ such that

$$
\mathscr{B} \subset \bigcap_{i=1}^{r}\left\{X \in \mathscr{L} ; X\left(a_{i}\right)=0\right\} .
$$

b) The formal algebra $B$ of $\mathscr{B}$ is equal to $L$ at every point of $M$. In particular, $\mathscr{B}$ is transitive at every point of $M$.

Proof. Let $B_{x}$ denote the formal algebra of $\mathscr{B}$ at $x \in M$. Put $S(\mathscr{B})=\{x \in M$; $\left.B_{x} \sqsubseteq L\right\}$. Since $\operatorname{dim} \mathscr{L} \mid \mathscr{B}<\infty, B_{x}$ is a Lie subalgebra of finite codimension of $L$, and $S(\mathscr{B})$ is at most a finite set. We first consider the case of $S(\mathscr{B})=\left\{a_{1}, \cdots, a_{r}\right\}$. In this case, each $B_{a_{i}}$ is a proper Lie subalgebra of finite codimension of $L$. (Each point $a_{i}$ is considered to be included in a certain $\mathscr{L}$-coordinate neighborhood.) Then by Theorems 3.1 and 7.1, we see that any $X \in \mathscr{B}$ vanishes at each $a_{i}$.

Note that even if $\mathscr{B}$ is proper, it may happen that $S(\mathscr{B})=\phi$. We give such an example of $\mathscr{B}$ and $\mathscr{L}$ hereinafter.

q.e.d.

We give an example of $\mathscr{B}$ and $\mathscr{L}$ which occur in b) of Theorem 7.2. Let $M$ be a compact symplectic manifold. Define a Lie subalgebra $\mathscr{B}$ of $\mathscr{L}_{s p}(M, \omega)$ by

$$
\mathscr{B}=\left\{X \in \mathscr{L}_{s p}(M, \omega) ; i(X) \omega=d f, f \text { is a function on } M\right\} .
$$

Then it is well-known that $\operatorname{dim} \mathscr{L}_{s p}(M, \omega) / \mathscr{B}=$ the betti number of $M$. But clearly $B=L_{s p}(2 n)$ at any point of $M$.

\section{§8. Remark (a counterexample)}

In $\S 3$ and $\S 7$, we have proved that any proper Lie subalgebra of finite codimension of the classical infinite Lie algebras is necessarily closed and is contained in $L_{0}$. But if $L$ is a general transitive infinite Lie algebra with a transitive filtration $L=\left\{L_{p}\right\}_{p \in Z}$, it may happen that $L$ admits a Lie subalgebra $B$ of finite codimension such that $B \not \subset L_{0}$. We give such an example.

Let $V$ be a 2-dimensional vector space with a basis $\partial / \partial x$ and $\partial / \partial y$. Put $\mathfrak{g}_{-1}=V$ and $g_{p}=\left\{x^{p+1}(\partial / \partial y)\right\}$ for $p \geqq 0$. Then we obtain a transitive infinite Lie algebra $L=\prod_{p \geqq-1} g_{p}$, which is the topological completion of the graded Lie algebra $\sum_{p \geqq-1} \mathfrak{g}_{p}$. Now $B=\partial / \partial y+\prod_{p \geqq 0} \mathfrak{g}_{p}$ is an abelian Lie subalgebra and is of codimension one. Clearly $B$ is not contained in $L_{0}=\prod_{p \geqq 0} \mathfrak{g}_{p}$. 


\section{References}

[ 1 ] S. Kobayashi and T. Nagano, On filtered Lie algebras and geometric structures, III and IV, J. Math. Mech., 14 (1965), 679-706, and 15 (1966), 163-175.

[2] K. Masuda, Homomorphism of the Lie algebras of vector fields, J. Math. Soc. Japan, Vol. 28, No. 3 (1976), 506-528.

[ 3 ] T. Morimoto, The derivation algebras of the classical infinite Lie algebras, J. Math. Kyoto Univ., Vol. 16, No. 1, (1976), 1-24.

[4] T. Morimoto and N. Tanaka, The classification of the real primitive infinite Lie algebras, J. Math. Kyoto Univ., 10 (1970), 207-243.

[5] N. Nakanishi, On the structure of infinite primitive Lie algebras, Proc. Japan Acad., Vol. 52, No. 1, (1976), 14-16.

[6] N. Nakanishi, Sous-algèbres de Lie de codimension finie de l'algèbre de Lie des transformations infinitésimales d'une structure de contact, C. R. Acad. Sc. Paris, t.283, (1976), 907-910.

[ 7 ] N. Nakanishi, Lie subalgebras of finite codimension in the restricted Poisson algebra, J. Math. Kyoto Univ., Vol. 17, No. 1, (1977), 27-34.

[ 8 ] I. M. Singer and S. Sternberg, On the infinite groups of Lie and Cartan, Part 1 (the transitive groups), J. Analyse Math., 15, (1965), 1-114. 